\title{
Reflets
}

Revue ontaroise d'intervention sociale et communautaire

\section{Le rôle de l'hôpital communautaire Montfort comme pilier francophone de formation}

\section{John Joanisse}

Volume 8, numéro 1, printemps 2002

La réadaptation : son visage français en Ontario

URI : https://id.erudit.org/iderudit/026382ar

DOI : https://doi.org/10.7202/026382ar

Aller au sommaire du numéro

Éditeur(s)

Reflets : Revue ontaroise d'intervention sociale et communautaire

ISSN

1203-4576 (imprimé)

1712-8498 (numérique)

Découvrir la revue

Citer cet article

Joanisse, J. (2002). Le rôle de l'hôpital communautaire Montfort comme pilier francophone de formation. Reflets, 8(1), 169-172.

https://doi.org/10.7202/026382ar

Tous droits réservés (C) Reflets : Revue ontaroise d'intervention sociale et communautaire, 2002
Ce document est protégé par la loi sur le droit d'auteur. L'utilisation des services d’Érudit (y compris la reproduction) est assujettie à sa politique d'utilisation que vous pouvez consulter en ligne.

https://apropos.erudit.org/fr/usagers/politique-dutilisation/ 


\title{
Le rôle de l'hôpital communautaire Montfort comme pilier francophone de formation
}

\author{
Dr. John Joanisse \\ Hôpital Montfort, Ottawa
}

\section{Le point de départ}

Une partie intégrale de la formation de tout professionnel comprend un élément de formation pratique. Son importance est d'autant plus critique dans une profession de soins de la santé telle que la réadaptation, les soins infirmiers et la médecine où il $\mathrm{y}$ a une interaction verbale et physique entre le professionnel et le patient.

Plusieurs milieux de santé sont en mesure d'offrir des environnements propices à l'apprentissage clinique, les avantages étant variés. Les prochains paragraphes exposeront les avantages de la formation pratique dans un hôpital communautaire en citant comme exemple l'Hôpital Montfort. La récente victoire de Montfort face aux menaces de fermeture et ses nouveaux locaux dédiés à la formation font de cette institution un modèle intéressant à explorer.

L'hôpital communautaire a comme mission d'identifier et de connaitre la communauté qu'il dessert. Son territoire d'impact est souvent établi selon des démarcations géographiques mais aussi selon des particularités démographiques telles la langue majoritaire de la population cible. L'hôpital communautaire doit créer des liens 
avec sa clientèle et répondre précisément aux besoins socioculturels pour devenir un partenaire intégral dans le continuum des services de santé. Les exemples concrets d'intégration sont les services ambulatoires offerts par des cliniques externes ou satellites. En retour, la communauté appuie activement son établissement de santé communautaire, osant même la défendre.

\section{La formation clinique}

Tous les enseignants cliniques dans le domaine de la santé sont appelés à modifier leur fonctionnement lorsqu'il est question de la formation clinique des étudiants. Pour nommer quelquesunes des modifications, on parle de réaménagement des espaces physiques, d'une réorganisation de la gestion du temps, d'une mise au point des connaissances et même des changements dans l'assignation des patients.

Alors pourquoi se dédier à l'enseignement clinique? Les bénéfices intangibles sont souvent les plus importants. L'occasion de côtoyer des futurs professionnels et de partager leur enthousiasme envers la profession et les patients ne peut se monnayer. L'enseignement clinique découle en partie du désir moral de remettre un peu de soi dans le système éducatif et de modeler l'aspect clinique des apprenants.

Les bénéfices tangibles quant à eux, se résument souvent à une reconnaissance formelle universitaire, une affiliation en quelque sorte. Il est également important de reconnaitre la possibilité de croissance personnelle, de modification ou d'acquisition de nouvelles compétences, par exemple en enseignement, et de changement dans les tâches de travail.

Du côté des étudiants, les avantages d'une expérience en milieu hospitalier communautaire sont nombreux. Le Rapport de l'Ontario Council of University Programs Rehabilitation Sciences de 2001, explique que les stages en communauté permettent un mentorat professionnel, une occasion de comprendre les conflits 
professionnels, une amélioration dans les habiletés cliniques et psychomotrices, une augmentation dans le niveau de confiance et la conscientisation aux déterminants de la santé. Bref,l'étudiant apprend à être à l'écoute de sa communauté. Il fait face à des expériences variées puisque la spécialité des hôpitaux communautaires c'est, en réalité, d'être généraliste. La prévention étant l'une des missions importantes que se donne tout hôpital communautaire, les étudiants ont également l'occasion de formellement appliquer ces principes.

Il est clair que pour l'hôpital communautaire, le plus grand bénéfice est d'être exposé à un bassin important de futurs employés capables de desservir sa communauté d'attache ou toute autre communauté ayant le même profil. En retour, l'hôpital communautaire se doit d'appuyer ses enseignants cliniques en tant que gestionnaires des ressources humaines et physiques, mais aussi en tant que facilitateurs du développement professionnel de ses employés.

\section{La formation professionnelle}

L'Hôpital communautaire doit permettre à ses employés et particulièrement à ses enseignants cliniques de parfaire leurs connaissances et compétences professionnelles. La formation professionnelle permet de mieux agir en tant que pourvoyeur de services en santé et aussi en tant qu'enseignant clinique.

L'Hôpital Montfort en collaboration avec Patrimoine Canada a rendu cette formation professionnelle accessible à ses employés à même les lieux physiques de l'hôpital. En effet, un espace important a été réservé pour le Centre national de formation en santé et le Bureau des affaires francophones de la faculté de médecine de l'Université d'Ottawa pour appuyer les efforts de formation.

Les professionnels et les étudiants de l'Hôpital Montfort ont maintenant un accès accru à des ressources technologiques, à de 
la formation à distance en ligne ou par vidéoconférence, à une bibliothèque autant physique que virtuelle, à une équipe de professionnels en Sciences de la santé dédiés à la formation et à des locaux aménagés spécifiquement pour l'enseignement professionnel et clinique. C'est le type d'appui académique qui est absolument nécessaire pour permettre aux cliniciens d'embrasser leur rôle de formateurs. L'Hôpital communautaire Montfort est donc devenu un pilier important de formation et d'enseignement.

De plus, il est maintenant possible pour l'Hôpital Montfort de s'allier aux autres hôpitaux communautaires canadiens desservant des populations cibles similaires pour échanger et partager les ressources en formation de langue française. On parle de présentations, de séminaires, de didacticiels ou d'autres outils de formation. Bien qu'un réseau étroit de formation entre les établissements de santé communautaire soit une vision de l'avenir, les moyens sont en place et leurs efforts reconnus par les institutions académiques post-secondaires.

Dorénavant, à l'Hôpital Montfort, les professions majoritaires telles les médecins et infirmières et de façon plus importante, les professions minoritaires, telles les kinésiologues, les physiothérapeutes, les ergothérapeutes, les orthophonistes et les audiologistes pourront profiter d'un appui physique et moral de leur employeur. Elles auront à apprendre à exploiter les ressources mises à leur disposition au profit de leur communauté qui a un grand besoin de professionnels francophones qualifiés pour la servir. Les étudiants en sciences de la santé sauront sûrement profiter des avantages que peuvent leur offrir les hôpitaux communautaires comme Montfort. 